\title{
Linguistic Intraoperative Protocol
}

\author{
Luis M.T. Jesus ${ }^{1,2}$, Joana Alves ${ }^{1}$, Mafalda Cardoso ${ }^{1}$, Mariana Morgado ${ }^{1}$ \\ ${ }^{1}$ School of Health Sciences (ESSUA), University of Aveiro, Portugal \\ ${ }^{2}$ Institute of Electronics and Informatics Engineering of Aveiro (IEETA), University of \\ Aveiro, Portugal \\ https://doi.org/10.36505/ExLing-2019/10/0030/000392
}

\begin{abstract}
The Dutch Linguistic Intraoperative Protocol - EP (DuLIP-EP) is a culturally adapted and validated instrument that allows language evaluation in patients with low-grade gliomas submitted to awake brain surgery. It assesses language areas which are related to different brain regions and pathways. In this paper, each task included in DuLIP-EP is presented and some preliminary results from its application to a normal Portuguese population are discussed.
\end{abstract}

Key words: language, brain, mapping, awake surgery

\section{Introduction}

The Dutch Linguistic Intraoperative Protocol (DuLIP) was originally developed in the Netherlands, to evaluate pre-, intra-, and post-operative language skills in patients with low-grade gliomas (De Witte et al., 2015). In Portugal, there are no validated instruments to assess language skills during surgery. This study aims to translate to European Portuguese (EP), culturally adapt and validate the DuLIP in the general population. This protocol includes phonological, semantics, syntax and articulation tasks, that are applied to the patients according to their tumour localisation and its functional implications.

\section{Methods and description of the tests}

The DuLIP-EP was initially based on a literal translation of the original tool, but the resulting materials were also adapted according to variables such as frequency, imageability, age of acquisition, prevalence and word class. Specific criteria, such as complexity levels, number of phonemes, number of syllables and syllabic structure, were used to build the final version of the repetition task. Furthermore, in the tasks that involve phrases, criteria such as time/verbal mode, order of constituents in the sentence, number of words, passive/active voice, were used.

To validate the final version of the protocol, test administration, registration and correction of the items were discussed by the three authors of this paper, particularly when uncertainties regarding the scoring, until a consensus was found.

ExLing 2019: Proceedings of 10 $0^{\text {th }}$ International Conference of Experimental Linguistics, 25-27 September 2019, Lisbon, Portugal 
122 L.M.T. Jesus et al.

Seventy (70) volunteers were randomly selected from a larger database for analysis (data collection still underway). Inclusion criteria were defined according to the original study (De Witte et al., 2015), but considering that native language should be EP.

Participants were 25 male and 45 females. Regarding age, two groups were created: $\geq 55$ years old $(n=12)$ and $\geq 18<55(n=68)$. Ages varied between 18 and 89, with an average (AVG) of 37.20 [standard deviation $(\mathrm{SD})=16.06$ ]. Fourteen (14) participants had less than 12 years of schooling (AVG=15.03; $\mathrm{SD}=4.10)$. Addenbrooke's Cognitive Examination scores varied between 76 and $100(\mathrm{AVG}=15.03 ; \mathrm{SD}=4,64)$.

In all tasks performed there is an intervention of various brain areas, so each assignment evaluates and stimulates different areas. Moreover, some tasks have a time frame so that Direct Electrical Stimulation (DES) can be used to assess the function role of restricted cortical and subcortical regions while in surgery.

\section{Phonology}

Phonology tasks include: Repetition of words (4s time frame) - This task aims to evaluate the ability to produce words according to their auditory discrimination. It contemplates 300 stimuli organised by different levels of complexity; Phonological odd word out (without time frame) - The objective of this task is to evaluate the phonological awareness. There are 15 lists with 4 monosyllabic words where participants should identify the intruder; Phonological sentence judgment - This task contemplates 30 sentences, which must be validated by the participants as being correct or incorrect. The incorrect ones are those containing non-words; Phonological fluency - This task evaluates the capacity of phonological evocation. In a minute, the participants should say the maximum number of words beginning with $/ \mathrm{m} /$, $/ \mathrm{p} /$ and $/ \mathrm{R} /$ phonemes.

\section{Semantics}

Testing of semantics is based on: Reading with semantic odd word out (4s time frame) - The participants must read aloud the word that doesn't fit semantically a list of 4 words (25 items); Reading with semantic odd picture out Participants have to name the picture that doesn't fit semantically in a group of 3 drawings (25 items); Semantic association

Participants should read two words and add a third semantically linked to the previous ones (25 items); Sentence completion (close context, $4 \mathrm{~s}$ time frame) Two tasks to evaluate this capacity. One is prompted by a specific context, the other evaluates speech production with an open context/less semantically induced. Participants must read out aloud and complete sentences in a meaningful way (25 items per task); Semantic sentence judgment (without time frame) - The goal of this task is to discriminate between semantically correct and incorrect sentences (50 items); Semantic fluency - Within a minute each, participants have to name as many animals and jobs as possible. 


\section{Syntax}

Syntax evaluation tasks include: Verb generation (4s time frame) - Singular nouns are presented in order and participants are asked to semantically relate them to a verb, evoking them; Syntactic sentence judgment (without time frame) - This task aims to assess the capacity to discriminate between 50 correct and incorrect sentences, syntactically speaking; Syntactic fluency During one minute, the subjects have to produce as many verbs as possible.

\section{Articulation}

Verbal diadochokinesis: This task includes 9 sequences that the participants should produce as fast and accurate as possible. This task should be timed.

Naming (4s time frame)

Object naming of 100 black and white drawings.

\section{Results}

Global phonology scores were: Repetition Task (AVG $=299.17$; $\mathrm{SD}=1.063)$, in a total of 300 items; Phonological Intruder Analysis (AVG = 14.56; SD =1.04), in a total of 15 items; Phonological Sentence Judgment $(\mathrm{AVG}=29.96$; $\mathrm{SD}=$ $0.20)$, in a total of 30 items; Phonological Fluency of $/ \mathrm{m} /, / \mathrm{R} /$ and $/ \mathrm{p} /$ phonemes $(\mathrm{AVG}=14.56 ; \mathrm{SD}=6.03),(\mathrm{AVG}=18.26 ; \mathrm{SD}=6.05)$ and $(\mathrm{AVG}$ $=18.90 ; \mathrm{SD}=6.86)$, respectively.

Semantics scores results were: Semantic Odd Word Out (AVG $=23.51 ;$ SD $=2.22)$ and Odd Picture Out $(\mathrm{AVG}=24.33 ; \mathrm{SD}=1.19)$, out of 25 pictures and words; Semantic Association (AVG $=21.81 ; \mathrm{SD}=2.42)$ in a total of 25 ; Semantic Completion with close context $(\mathrm{AVG}=24.50 ; \mathrm{SD}=1.09)$, lower scores when compared with the less induced context $(\mathrm{AVG}=24.93 ; \mathrm{SD}=$ 0.31 ), each one with 25 items. Semantic Sentence Judgment (AVG = 48.89; $\mathrm{SD}=1.40)$, in a total of 50 items; Semantic Fluency: animals and jobs (AVG = 24.17; $\mathrm{SD}=6.05)$ and $(\mathrm{AVG}=20.06, \mathrm{SD}=5.24)$, respectively.

Syntactic tasks scores were: Verb Generation $(\mathrm{AVG}=47.14 ; \mathrm{SD}=4.37)$ in a total of 50; Syntactic Sentence Judgement I \& II (AVG = 24.54; SD = 0.63) and $(\mathrm{AVG}=24.61 ; \mathrm{SD}=0.77)$, respectively, each with a total of 25 items; Syntactic fluency (AVG $=24.66 ; \mathrm{SD}=7.82$ ).

Object naming of 100 pictures average scores were 96.37 with a standard deviation of 3.65.

Diadochokinesis scores for each task were: Task $1-\mathrm{AVG}=3.03$; $(\mathrm{SD}=$ 1.30); Task $2-\mathrm{AVG}=2.07(\mathrm{SD}=1.04)$; Task $3-\mathrm{AVG}=2.78$; $(\mathrm{SD}=0.93)$; Task $4-\mathrm{AVG}=3.25(\mathrm{SD}=1.00)$ Task $5-\mathrm{AVG}=5.50(\mathrm{SD}=1.62)$; Task $6-$ $\mathrm{AVG}=7.44(\mathrm{SD}=2.72) ;$ Task $7-\mathrm{AVG}=6.06(\mathrm{SD}=2.38) ;$ Task $8-\mathrm{AVG}=$ $6.52(\mathrm{SD}=2.15)$; Task $9-\mathrm{AVG}=6.82(\mathrm{SD}=2.19)$. 
124 L.M.T. Jesus et al.

\section{Conclusions}

In general, most of the tasks have a mean score close to the maximum score desired which could indicate that the tasks are well tailored and well perceived by the participants. Yet, some adjustments are needed. Since this was a preliminary test, the SD values were higher than desirable, but we aim to expand testing to 140 participants in order to reach more solid results. As future work it would be useful to apply the DuLIP-EP protocol to some clinical cases as well study possible correlations between variables.

\section{Acknowledgements}

The authors would like to thank Evy Brink, Djaina Satoer and Elke Witte. This research was funded by the Foundation for Science and Technology (FCT), in the context of the project UID/CEC/00127/2019.

\section{References}

De Witte, E., Satoer, D., Robert, E., Colle, H., Verheyen, S., Visch-Brink, E., Mariën, P. 2015. The Dutch Linguistic Intraoperative Protocol: A valid linguistic approach to awake brain surgery. Brain and Language, 140, 35-48. 